\title{
Experiencia del uso de polvo hemostático en el manejo endoscópico de sangrado variceal y no variceal en un hospital de tercer nivel en México. El primer reporte en pacientes pediátricos en Latinoamérica
}

\author{
José Cadena-León ${ }^{1}$, Jaime Alfaro-Bolaños², Luz Tovar-Correa², Mayra Medranda-Cedeño², \\ Monserrat Cázares-Méndez ${ }^{1}$, Ericka Montijo-Barrios ${ }^{1}$, Flora Zárate-Mondragón², Erick Toro-Monjaraz², \\ Karen Ignorosa-Arellano², Roberto Cervantes ${ }^{2}$ y Jaime Ramírez-Mayans ${ }^{3}$ \\ ${ }^{1}$ Unidad de Endoscopia, Departamento de Gastroenterología y Nutrición Pediátrica; ${ }^{2}$ Departamento de Gastroenterología y Nutrición Pediátrica; \\ ${ }^{3}$ Unidad Diagnóstica Gastroenterología de Pediátrica Integral, Departamento de Gastroenterología y Nutrición Pediátrica. Instituto Nacional de \\ Pediatría, Ciudad de México, México
}

\begin{abstract}
Resumen
El sangrado de tubo digestivo es una condición que amenaza la vida en la edad pediátrica y es una de las principales indicaciones para realizar una endoscopia diagnóstica y terapéutica. A pesar de que hay múltiples técnicas para el manejo tanto de la mortalidad como de la recurrencia del sangrado, esta aún sigue siendo muy alta en la infancia. Actualmente se ha propuesto el uso de polvo hemostático como una alternativa práctica y eficaz para el manejo del sangrado de tubo digestivo. Objetivo: Describir la experiencia del uso de polvo hemostático como tratamiento endoscópico alternativo para sangrado variceal y no variceal en pacientes pediátricos. Material y métodos: Estudio retrospectivo, observacional y descriptivo, de pacientes pediátricos de 1-18 años de edad con sangrado de tubo digestivo alto y bajo, en quienes se aplicó polvo hemostático como tratamiento alternativo del sangrado en el Instituto Nacional de Pediatría entre agosto de 2017 a noviembre de 2018. Resultados: Se incluyeron 10 pacientes pediátricos con sangrado de tubo digestivo tanto alto como bajo de diferentes causas, en los cuales el sangrado fue anemizante. Se aplicó Hemospray ${ }^{\circ}$, lográndose control del sangrado en el $90 \%$ de los pacientes con una aplicación. Conclusión: El uso se Hemospray constituye una alternativa fácil y rápida con buenos resultados para el control de sangrado de tubo digestivo tanto variceal como no variceal.
\end{abstract}

Palabras clave: Endoscopia. Sangrado de tubo digestivo. Pediatría. Hemospray.

\section{Correspondencia:}

*José Cadena-León

Insurgentes Sur, 3700, Letra C

Del. Coyoacán

Fecha de recepción: 01-02-2019

C.P. 04530, Ciudad de México, México

E-mail: pcaden60@ hotmail.com
Disponible en internet: 06-09-2019

Endoscopia. 2019;31:125-129

www.endoscopia-ameg.com

0188-9893/๑ 2019. Asociación Mexicana de Endoscopia Gastrointestinal, publicado por Permanyer México SA de CV, todos los derechos reservados. 


\title{
Experience of the use of hemostatic powder in the endoscopic management of variceal and non-variceal bleeding in a tertiary hospital in Mexico. The first report in pediatric patients in Latin America
}

\begin{abstract}
Gastrointestinal bleeding is a life-threatening condition in the pediatric age and is one of the main indications for diagnostic and therapeutic endoscopy, although there are multiple techniques for managing both mortality and recurrence of bleeding being very high in childhood. Currently, the use of hemostatic powder has been proposed as a practical and effective alternative for the management of digestive tract bleeding. Objective: To describe the experience of the use of hemostatic powder as an alternative endoscopic treatment for variceal and non-variceal gastrointestinal bleeding in pediatric patients. Material and methods: Retrospective, observational and descriptive study of pediatric patients aged 1-18 years with upper and lower gastrointestinal bleeding, in whom hemostatic powder was applied as an alternative treatment of bleeding at the National Institute of Pediatrics between January 2017 and November 2018. Results: We included 10 pediatric patients with upper and lower gastrointestinal bleeding from different causes, in whom the bleeding was anemic. Hemospray was applied, achieving control of bleeding in $90 \%$ of patients with one application. Conclusion: The use of Hemospray is an easy and fast alternative with good results for the control of both variceal and non-variceal gastrointestinal bleeding.
\end{abstract}

Key words: Endoscopy. Gastrointestinal bleeding. Pediatrics. Hemospray.

\section{Introducción}

La hemorragia de tubo digestivo (HTD) es una de las entidades clínicas con mayor morbimortalidad en pediatría. Se divide en tres presentaciones clínicas: HTD alta, HTD baja y sangrado de origen oscuro. La etiología de la HTD depende de la edad del niño. En el recién nacido, las causas más frecuentes son malformación arteriovenosa, úlceras gástricas, alteración de la coagulación, alergia a la proteína de leche y enterocolitis necrosante. En lactantes se puede encontrar además divertículo de Meckel, diarrea infecciosa y lesión anorrectal. En el grupo de escolares y adolescentes, esofagitis, gastropatías, várices esofágicas y/o gástricas, pólipos, hiperplasia nodular linfoide y enfermedad inflamatoria intestinal (EII) entre otras ${ }^{1,2}$. En el enfoque inicial es importante determinar la severidad del sangrado como anemizante o no anemizante. La HTD anemizante se refiere a una pérdida de $15-20 \%$ o más del volumen sanguíneo circulante, una disminución mayor o igual a $2 \mathrm{~g} / \mathrm{dl}$ de la hemoglobina con 0 sin compromiso del estado hemodinámico. Usualmente este tipo de sangrado es el que nos obliga a un diagnóstico y tratamiento tempranos ${ }^{3}$.

El uso de polvo hemostático TC-325 (Hemospray ${ }^{\circledR}$ Cook medical, EE.UU.) es un dispositivo nuevo para hemostasia endoscópica que inicialmente fue creado para su utilización en el sangrado gastrointestinal no variceal. Al entrar en contacto dicho polvo con la sangre 0 el tejido en el tracto gastrointestinal se adhiere formando una barrera mecánica que cubre el sitio de sangrado, logrando una hemostasia muy rápida 4 .
Holster, et al. realizaron un estudio in vitro e in vivo para determinar el mecanismo y tiempo de acción del TC-325, en el que realizaron diferentes pruebas como tiempo de recalcificación, tromboelastograma y tiempos de coagulación, concluyendo que tiene un tiempo de inicio de coagulación de 45-60 s $(p<0.05)^{5}$.

El TC-325 consiste en un polvo inorgánico que al entrar en contacto con líquidos o soluciones viscosas, como por ejemplo sangre, se adhiere y forma un tapón, cubriendo así el sitio de sangrado. Por medio de la absorción del líquido o la sangre, permite de esta manera que aumente la concentración de factores de coagulación y activa la cascada de la coagulación ${ }^{6}$. Aproximadamente después de $24 \mathrm{~h}$ la capa adherente que se formó se va desprendiendo en el lumen intestinal y es eliminada del tracto gastrointestinal ${ }^{4}$.

Dentro de las principales indicaciones para su uso se encuentran lesiones de difícil acceso endoscópico, hemorragia gastrointestinal masiva, múltiples sitios de sangrado, modificación de la anatomía por terapia endoscópica previa, presencia de coagulopatía, dificultad para tener visualización directa o cuando es imposible tener contacto con la lesión sangrante?

\section{Objetivo}

Describir la experiencia del uso de polvo hemostático como tratamiento endoscópico alternativo para sangrado variceal y no variceal en pacientes pediátricos en el Instituto Nacional de Pediatría. 


\section{Material y métodos}

Estudio retrospectivo, observacional y descriptivo. Incluimos a pacientes pediátricos (1 a 18 años de edad) que presentaron HTD en quienes se utilizó Hemospray ${ }^{\circledR}$ (HEMO 7, de 7 fr y catéter con longitud de $220 \mathrm{~mm}$ ) como tratamiento del sangrado; en todos los casos se utilizaron endoscopios pediátricos Olympus GIF-Q150 ${ }^{\circledR}$. Se revisó la base de datos electrónica del Servicio de Endoscopia del Instituto Nacional de Pediatría, de enero de 2017 a diciembre de 2018. Se describen variables sociodemográficas, el procedimiento realizado, y la localización, el tipo, la etiología y la resolución del sangrado.

El estudio se apega a los principios de publicación del Comité de Ética del Instituto Nacional de Pediatría y cumple con la declaración de los principios de Helsinki.

\section{Resultados}

Se incluyeron 10 pacientes con HTD en quienes se aplicó Hemospray ${ }^{\circledR}$. La edad media fue de 76 meses (rango: 17-215 meses). Siete pacientes eran del sexo masculino y tres femeninos.

Tres pacientes se encontraban en la Unidad de Cuidados Intensivos, con inestabilidad hemodinámica. Todos los pacientes presentaron sangrado anemizante, definido por un descenso mayor o igual a $2 \mathrm{~g} / \mathrm{dl}$ de hemoglobina respecto a la basal y cinco presentaban coagulopatía, definida como INR > 1.2.

Los diagnósticos de base de los pacientes incluidos en el presente estudio fueron: hipertensión portal $(n=3)$, enfermedad injerto contra huésped $(E I C H)$ intestinal $(n=2)$, falla multisistémica $(n=1)$, sin diagnostico $(n=1)$, Ell y probable displasia hipodérmica hipohidrótica $(n=1)$, leucemia linfoide aguda $(n=1)$ y rabdomiosarcoma pélvico $(n=1)$. En nueve pacientes solo se realizó una aplicación, obteniendo buenos resultados, ya que el sangrado se logró controlar $(90 \%$ de los casos), excepto en un caso en que el sangrado fue recurrente y se tuvo que aplicar en dos ocasiones diferentes el Hemospray ${ }^{\circledR}$ (Tabla 1).

\section{Discusión}

El Hemospray ${ }^{\circledR}$ forma parte del tratamiento del sangrado de tubo digestivo alto. En un inicio se propuso para ser utilizado como manejo alternativo del sangrado recurrente secundario a úlceras pépticas que no respondían al tratamiento que habitualmente se utiliza, como argón plasma, hemoclips o inyección de adrenalina, entre otros ${ }^{4}$. En dichos casos se han obtenido buenos resultados, logrando hemostasia hasta en el $95 \%$ de los pacientes en un estudio prospectivo de 20 pacientes en donde en el $85 \%$ se utilizó una sola aplicación y en el $15 \%$, dos aplicaciones ${ }^{8}$. En general se ha demostrado en diferentes estudios reportados en adultos una tasa de efectividad de entre el 93 y el $98 \%$ en el control del sangrado ${ }^{9-11}$.

Recientemente se empezó a utilizar Hemospray ${ }^{\circledR}$ en México, que ha sido el primer país en Latinoamérica en utilizarlo. Actualmente en niños la experiencia es poca, pero cada vez se reportan más estudios de la efectividad del Hemospray ${ }^{\circledR}$, no solo en sangrado variceal sino también en sangrado variceal y gastropatía portal ${ }^{12,13}$.

Hay reportes de la utilización de TC-325 en pacientes por diferentes razones, entre las que se mencionan: úlceras pépticas, tumores sangrantes, papilomatosis, esofagitis secundaria a reflujo, síndrome de Mallory-Weiss, várices esofágicas y gástricas, lesiones de Dieulafoys, úlceras duodenales y gástricas, etc. ${ }^{14-17}$. En nuestro instituto contamos con el Hemospray ${ }^{\circledR}$ desde hace un poco más de un año y se ha utilizado en diferentes circunstancias, como lo reporta la literatura internacional. Se han obtenido resultados: en el $90 \%$ de los casos se logró control del sangrado de tubo digestivo alto y bajo, y solo en el $10 \%$ (un paciente) no se logró controlarlo y se requirieron dos intervenciones en diferentes momentos. Cabe mencionar que este paciente tenía colitis neutropénica, por lo cual fue sometido a colectomía y a ileoctomía; finalmente dicho paciente falleció por complicaciones infecciosas. Los resultados de nuestro trabajo son similares a los demostrados en otros estudios en pacientes en la edad adulta $^{8}$.

Hasta la fecha se ha considerado el uso de Hemospray ${ }^{\circledR}$ como una medida segura para control de la HTD tanto variceal como no variceal. El Hemospray ${ }^{\circledR}$ es fácil de usar y se asocia con pocos efectos adversos. Tiene muchas ventajas, como su practicidad, ya que una vez identificada la lesión o sitio de sangrado solo se pasa de manera simultánea aire con una jeringa de $50 \mathrm{ml}$, tanto por el canal de trabajo del endoscopio como por el catéter del Hemospray ${ }^{\circledR}$ de manera simultánea para luego descargar el polvo, con accesibilidad a lesiones que son de difícil acceso endoscópico para poder realizar alguna técnica hemostática convencional; otra ventaja es la habilidad para tratar una superficie amplia de la mucosa gastrointestinal. A pesar de que se recomienda utilizar el dispositivo en solo una ocasión, en 
Tabla 1. Resultados del estudio

\begin{tabular}{|c|c|c|c|c|c|c|c|c|c|}
\hline N. ${ }^{\circ}$ & Sexo & $\begin{array}{c}\mathrm{N}^{\circ}{ }^{\circ} \\
\text { aplicaciones }\end{array}$ & $\begin{array}{c}\text { Edad } \\
\text { (meses) }\end{array}$ & Procedimiento & Diagnóstico & $\begin{array}{l}\text { Administración } \\
\text { Hemospray }\end{array}$ & $\begin{array}{l}\text { Control del } \\
\text { sangrado }\end{array}$ & $\begin{array}{l}\text { Endoscopio } \\
\text { usado }\end{array}$ & $\begin{array}{l}\text { Modelo } n^{0} \\
\text { sonda del } \\
\text { TC-325 }\end{array}$ \\
\hline 1 & $M$ & 1 & 80 & Panendoscopia & $\begin{array}{l}\text { HTP, post Warren, } \\
\text { posligadura de } \\
\text { varices esofágicas } \\
\text { grado II según } \\
\text { clasificación } \\
\text { japonesa }\end{array}$ & $\begin{array}{l}\text { Curvatura menor y } \\
\text { fondo por gastropatía } \\
\text { hemorrágica }\end{array}$ & Sí & $\begin{array}{l}\text { Olympus } \\
\text { GIF-0150 }\end{array}$ & $\begin{array}{l}\text { Hemo-7 } \\
\text { Sonda } 7 \mathrm{fr}\end{array}$ \\
\hline 2 & $M$ & 2 & 215 & $\begin{array}{l}\text { Panendoscopia } \\
\text { Ileoscopia }\end{array}$ & $\begin{array}{l}\text { LLA, EICH, colitis } \\
\text { neutropénica, } \\
\text { infección por E. Coli }\end{array}$ & $\begin{array}{l}\text { Duodeno por } \\
\text { bulboduodenitis } \\
\text { erosiva severa y en } \\
\text { úlcera ileal }\end{array}$ & No & $\begin{array}{l}\text { Olympus } \\
\text { GIF-0150 }\end{array}$ & $\begin{array}{l}\text { Hemo-7 } \\
\text { Sonda } 7 \mathrm{fr}\end{array}$ \\
\hline 3 & $M$ & 1 & 23 & Panendoscopia & $\begin{array}{l}\text { Falla multisistémica, } \\
\text { probable úlcera por } \\
\text { estrés, desnutrición } \\
\text { moderada }\end{array}$ & $\begin{array}{l}\text { Úlcera en duodeno } \\
\text { IIA, IIB de la } \\
\text { clasificación de } \\
\text { Forrest }\end{array}$ & Sí & $\begin{array}{l}\text { Olympus } \\
\text { GIF-0150 }\end{array}$ & $\begin{array}{l}\text { Hemo-7 } \\
\text { Sonda } 7 \mathrm{fr}\end{array}$ \\
\hline 4 & $M$ & 1 & 130 & Panendoscopia & $\begin{array}{l}\text { HTD alta con } \\
\text { histopatología de } \\
\text { Helicobacter pylori } \\
\text { sin otro test positivo }\end{array}$ & $\begin{array}{l}\text { Fondo y cuerpo } \\
\text { gástrico por } \\
\text { gastropatía } \\
\text { hemorrágica }\end{array}$ & Sí & $\begin{array}{l}\text { Olympus } \\
\text { GIF-0150 }\end{array}$ & $\begin{array}{l}\text { Hemo-7 } \\
\text { Sonda } 7 \mathrm{fr}\end{array}$ \\
\hline 5 & $\mathrm{~F}$ & 1 & 54 & Panendoscopia & $\begin{array}{l}\text { HTP, post Warren, } \\
\text { várices esofágicas } \\
\text { grado III según } \\
\text { clasificación } \\
\text { japonesa, varices } \\
\text { gástricas IGV2, } \\
\text { cirrosis biliar } \\
\text { secundaria a atresia } \\
\text { de vías biliares }\end{array}$ & $\begin{array}{l}\text { Fondo, cuerpo y } \\
\text { antro por } \\
\text { pangastropatía } \\
\text { congestiva severa } \\
\text { con sangrado activo }\end{array}$ & Sí & $\begin{array}{l}\text { Olympus } \\
\text { GIF-0150 }\end{array}$ & $\begin{array}{l}\text { Hemo-7 } \\
\text { Sonda } 7 \mathrm{fr}\end{array}$ \\
\hline 6 & $\mathrm{M}$ & 1 & 72 & Panendoscopia & $\begin{array}{l}\text { Rabdomiosarcoma } \\
\text { pélvico de células } \\
\text { pequeñas con } \\
\text { metástasis a columna } \\
\text { vertebral, HTD alta }\end{array}$ & $\begin{array}{l}\text { Esófago inferior por } \\
\text { esofagitis erosiva y } \\
\text { en antro por úlcera } \\
\text { gástrica con } \\
\text { sangrado activo, } \\
\text { grado I clasificación } \\
\text { de Forrest }\end{array}$ & Sí & $\begin{array}{l}\text { Olympus } \\
\text { GIF-0150 }\end{array}$ & $\begin{array}{l}\text { Hemo-7 } \\
\text { Sonda } 7 \mathrm{fr}\end{array}$ \\
\hline 7 & $M$ & 1 & 24 & $\begin{array}{l}\text { Panendoscopia } \\
\text { Colonoscopia }\end{array}$ & $\begin{array}{l}\text { Displasia ectodérmica } \\
\text { hipohidrótica con } \\
\text { inmunodeficiencia } \\
\text { con probable } \\
\text { enfermedad } \\
\text { inflamatoria intestinal } \\
\text { tipo colitis ulcerativa, } \\
\text { sangrado de tubo } \\
\text { digestivo bajo }\end{array}$ & $\begin{array}{l}\text { Pancolitis ulcerativa. } \\
\text { Índice de Mayo para } \\
\text { colitis ulcerosa grado } \\
3 \text { (rectosigmoides y } \\
\text { colon descendente) }\end{array}$ & Sí & $\begin{array}{l}\text { Olympus } \\
\text { GIF-0150 }\end{array}$ & $\begin{array}{l}\text { Hemo-7 } \\
\text { Sonda } 7 \mathrm{fr}\end{array}$ \\
\hline 8 & $M$ & 1 & 60 & Panendoscopia & $\begin{array}{l}\text { Leucemia } \\
\text { linfoblástica aguda, } \\
\text { mucositis III, } \\
\text { coagulopatía }\end{array}$ & $\begin{array}{l}\text { Fondo y cuerpo } \\
\text { gástrico por } \\
\text { pangastropatía } \\
\text { hemorrágica severa } \\
\text { con sangrado activo }\end{array}$ & Sí & $\begin{array}{l}\text { Olympus } \\
\text { GIF-0150 }\end{array}$ & $\begin{array}{l}\text { Hemo-7 } \\
\text { Sonda } 7 \mathrm{fr}\end{array}$ \\
\hline 9 & $F$ & 1 & 17 & Panendoscopia & $\begin{array}{l}\text { HTP, cirrosis biliar } \\
\text { secundaria a AVB, } \\
\text { várices esofágicas } \\
\text { grado III clasificación } \\
\text { japonesa }\end{array}$ & $\begin{array}{l}\text { Esófago inferior } \\
\text { posterior a ligadura } \\
\text { de varices grado III } \\
\text { de clasificación } \\
\text { japonesa }\end{array}$ & Sí & $\begin{array}{l}\text { Olympus } \\
\text { GIF-0150 }\end{array}$ & $\begin{array}{l}\text { Hemo-7 } \\
\text { Sonda } 7 \mathrm{fr}\end{array}$ \\
\hline 10 & $F$ & 1 & 93 & Colonoscopia & $\begin{array}{l}\text { Anemia de Fanconi, } \\
\text { enfermedad de injerto } \\
\text { contra el huésped }\end{array}$ & $\begin{array}{l}\text { Recto por } \\
\text { proctocolitis } \\
\text { ulcerativa con } \\
\text { sangrado activo }\end{array}$ & Sí & $\begin{array}{l}\text { Olympus } \\
\text { GIF-0150 }\end{array}$ & $\begin{array}{l}\text { Hemo-7 } \\
\text { Sonda } 7 \mathrm{fr}\end{array}$ \\
\hline
\end{tabular}


nuestro hospital lo hemos utilizado más de una ocasión, ya que tenemos la ventaja de que el catéter del TC-325 tiene una longitud de $220 \mathrm{~mm}$ y en el caso de que la punta se llegue a tapar por el contacto con líquidos o sangre hemos cortado la punta y mandado a esterilizar el catéter. Sin embargo en otros casos no se ha podido utilizar más de una ocasión, ya que el dispositivo de disparo funciona con $\mathrm{CO}_{2}$ y muchas veces este se puede escapar, ya no logrando utilizarlo en otra ocasión.

\section{Conclusiones}

El uso de Hemospray ${ }^{\circledR}$ para la hemorragia digestiva ha tenido buenos resultados, alcanzando tasas de hemostasia arriba del $90 \%$ y con baja recurrencia, aunque inicialmente su principal indicación era para úlceras pépticas, rápidamente se ha utilizado para múltiples causas de sangrado en diferentes instituciones, como se reporta en la literatura internacional. Esto incluye el sangrado variceal, por lo que se puede proponer como una medida de rescate en aquellos casos en los que posteriormente a la ligadura o escleroterapia el paciente presenta hemorragia importante. En nuestra experiencia no tuvimos problemas para aplicar el polvo hemostático, se realizó sin complicaciones, ya que solo se necesita de un médico entrenado en endoscopia, a diferencia de otras técnicas hemostáticas como hemoclips, argón plasma e inyección de adrenalina, entre otras, que si requieren un entrenamiento exhaustivo. A la fecha son pocas las cohortes de los diferentes estudios. Se estima importante continuar con más estudios, especialmente en la edad pediátrica. Consideramos que el presente estudio puede ser un punto de partida para futuros estudios en el resto de Latinoamérica, ya que es uno de los primeros reportes del uso de Hemospray ${ }^{\circledR}$ en pacientes pediátricos que se realiza en Latinoamérica.

\section{Financiamiento}

No se recibió financiamiento de ningún tipo para la realización de este artículo.

\section{Conflicto de interés}

Los autores declaran no tener conflicto de interés.

\section{Bibliografía}

1. Jovel-Banegas LE, Cadena-León JF, Cázares-Méndez JM, Ramírez-Mayans JA, Cervantes-Bustamente R, Zárate-Mondragón FE, et al. Sangrado del tubo digestivo en pediatría. Diagnóstico y tratamiento. Acta Pediatr Mex. 2013;34:280-7.

2. Almanza-Miranda $E$, Peña Vélez $R$, Castañeda Ortiz RA, Chávez Aguilar LA, Zaragoza Arevalo GR, Gómez Navarro G. Degeneración cavernomatosa de la vena porta: experiencia de tratamiento médico y endoscópico en pacientes pediátricos del centro médico nacional 20 de noviembre. Endoscopia. 2017;29(1):29-34.

3. Jovel-Banegas L, Cadena-León J, Cázares-Méndez M. Sangrado de tubo digestivo en pediatría. Diagnóstico y tratamiento. Acta Pediatr Mex. 2013;34:280-7.

4. Sung JJ, Luo D, Wu JC, Ching JY, Chan FK, Lau JY, et al. Early clinical experience of the safety and effectiveness of Hemospray in achieving hemostasis in patients with acute peptic ulcer bleeding. Endoscopy. 2011;43:291-5.

5. Holster IL, van Beusekom HM, Kuipers EJ, Leebeek FW, de Maat MP, Tjwa ET. Effects of a hemostatic powder hemospray on coagulation and clot formation. Endoscopy. 2015;47(7):638-45.

6. Cahyadi O, Bauder M, Meier B, Caca K, Schmidt A. Effectiveness of TC-325 (Hemospray) for treatment of diffuse or refractory upper gastrointestinal bleeding - a single center experience. Endosc Int Open. 2017;5(11):E1159-64.

7. Martínez-Alcalá A, Mönkemüller K. Emerging endoscopy treatment for nonvariceal, upper gastrointestinal hemorrage. Gastrointest Endosc Clin N Am. 2018;28(3):307-20.

8. Yau AH, Ou G, Galorport C, Amar J, Bressler B, Donnellan F, et al. Safety and efficacy of Hemospray $(R)$ in upper gastrointestinal bleeding. Can J Gastroenterol Hepatol. 2014;28:72-6.

9. Chen YI, Barkun A, Nolan S. Hemostatic powder TC-325 in the management of upper and lower gastrointestinal bleeding: a two-year experience at a single institution. Endoscopy. 2015;47:167-71.

10. Sulz MC, Frei R, Meyenberger C, Bauerfeind P, Semadeni GM, Gubler C. Routine use of Hemospray for gastrointestinal bleeding: prospective two-center experience in Switzerland. Endoscopy. 2014;46:619-24.

11. Gralnek IM, Dumonceau JM, Kuipers EJ, Lanas A, Sanders DS, Kurien $\mathrm{M}$, et al. Diagnosis and management of nonvariceal upper gastrointestinal hemorrhage: European Society of Gastrointestinal Endoscopy (ESGE) Guideline. Endoscopy. 2015;47:a1-46.

12. Smith LA, Morris AJ, Stanley AJ. The use of hemospray in portal hypertensive bleeding; a case series. J Hepatol. 2014;60(2):457-60.

13. Ibrahim M, El-Mikkawy A, Abdel Hamid M, Abdalla H, Lemmers A, Mostafa I,et al. Early application of haemostatic powder added to standard management for oesophagogastric variceal bleeding: a randomised trial. Gut. 2019;68(5):844-53.

14. Yau AH, Ou G, Galorport C, Amar J, Bressler B, Donnellan F, et al. Safety and efficacy of Hemospray $(R)$ in upper gastrointestinal bleeding. Can J Gastroenterol Hepatol. 2014;28:72-6.

15. Cahyadi O, Bauder M, Meier B, Caca K, Schmidt A. Effectiveness of TC-325 (Hemospray) for treatment of diffuse or refractory upper gastrointestinal bleeding - a single center experience. Endosc Int Open. 2017; 5(11):E1159-64.

16. Kosasih S, Jalihal A, Chong VH. Maximizing the use of Hemospray. Indian J Gastroenterol. 2018;37(5):467-8.

17. Pittayanon R, Rerknimitr R, Barkun A. Prognostic factors affecting outcomes in patients with malignant Gl bleeding treated with a novel endoscopically delivered hemostatic powder. Gastrointest Endosc. 2018; 87(4):994-1002. 